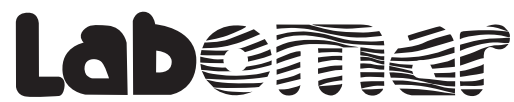

Arquivos de Ciências do Mar

\section{VALORAÇÃO AMBIENTAL DO PARQUE ESTADUAL MARINHO DA PEDRA DA RISGA DO MEIO, GEARÁ, BRASIL}

\author{
Environmental valuation of Pedra da Risca do \\ Meio Marine State Park, Ceará, Brazil \\ Pedro Bastos de Macedo Carneiro', Inah Sátiro², Clara de Melo $\mathrm{Coe}^{3}$, \\ Kamila Vieira de Mendonça ${ }^{4}$ \\ 1 Biólogo, Instituto de Ciências do Mar, Universidade Federal do Ceará, pedrocarneiro@ufc.br \\ 2 Doutoranda em Ciências Marinhas Tropicais, Universidade Federal do Ceará, inahsatiro@gmail.com \\ ${ }^{3}$ Mestranda em Engenharia de Pesca, Universidade Federal do Ceará, claracoe@yahoo.com.br \\ ${ }^{4}$ Professora Adjunta, Instituto de Ciências do Mar, Universidade Federal do Ceará, kamilavm@terra.com.br
}

\begin{abstract}
RESUMO
O estabelecimento de unidades de conservação é estratégia eficiente para a conservação da natureza, porém muitas dessas áreas são geridas sem critérios objetivos de alocação de recursos, o que muitas vezes resulta em aportes insuficientes. Buscando contribuir para a gestão de unidades de conservação marinhas do Brasil, o presente trabalho visa aplicar o método de valoração contingente para estimar valores de uso e não uso do Parque Estadual Marinho da Pedra da Risca do Meio, localizado no Ceará. Foram realizadas 105 entrevistas com moradores de Fortaleza, Ceará, e a influência de variáveis sociológicas (sintetizadas em estatísticas descritivas) sobre a disposição a pagar (DAP) pela manutenção do Parque foi estudada via modelos lineares. A disposição a pagar total foi calculada multiplicando a DAP pela população envolvida. Em média, os entrevistados se dispuseram a pagar $\mathrm{R}$ \$21,50/mês para a manutenção do Parque, valor que foi maior no caso de frequentadores e de pessoas de maiores rendas. Considerando apenas frequentadores, o valor de uso equivale a R \$ 964.080/ano. Esses resultados evidenciam a importância dessa unidade de conservação e ressaltam a necessidade de novas estratégias de financiamento para a sua manutenção. Maiores incentivos à visitação, cobrança de taxas e o pagamento por serviços ambientais prestados parecem ser mecanismos bem aceitos pela população local, além de poderem ser igualmente aplicados em outras unidades de conservação marinhas do Brasil, e portanto podem contribuir para a conservação da zona costeira do país.
\end{abstract}

Palavras-chave: gestão de unidade de conservação, valores de uso e não-uso, valoração contingente.

Recebido: 19 nov 2015

Aceito: 23 mar 2017

Publicado online: 31 mai 2017 


\section{ABSTRACT}

The establishment of protected areas is an efficient way to ensure nature conservation, but these areas are frequently managed without objective criteria for resource allocation, what often results in insufficient funding. In order to contribute to the management of marine protected areas in Brazil, this paper aims to estimate useand non-use values of Parque Estadual Marinho da Pedra da Risca do Meio, located in Ceará state, through the contingent valuation method. One hundred and five (105) interviews were conducted with residents of Fortaleza, Ceara, and the influence of sociological variables (summarized by descriptive statistics) on the Willingness To Pay (WTP) for the maintenance of the Park was studied through linear models. Total willingness to pay was calculated by multiplying WTP by the involved population. On average, respondents were willing to expend $R \$ 21.50$ / month for the maintenance of the Park, an amount that was higher in the case of users and people of higher incomes. Considering only users, the use value amounts to $R \$ 964.080$ / year. These results highlight the importance of this protected area and emphasize the need of new financing strategies for its maintenance. Increased incentives for visitation, collection of fees and the payment for environmental services seem to be well-accepted financing mechanisms, which are equally applicable to other Brazilian marine protected areas, and therefore can contribute to the conservation of the country's coastal zone.

Keywords: protected area management, use and non-use values, contingent valuation.

\section{INTRODUÇÃO}

O estabelecimento de unidades de conservação é amplamente visto como estratégia eficiente para a conservação da natureza. Ao longo das últimas décadas, as justificativas e os objetivos da criação dessas áreas mudou, passando da delimitação de santuários com elevada beleza cênica, voltados ao turismo contemplativo, para a defesa de sistemas críticos para manutenção da vida e de serviços ambientais, tendo em mente valores ecológicos, econômicos e sociais (Ervin et al., 2010).

Essa mudança de paradigmas vem, necessariamente, acompanhada de mudanças nas estratégias de gestão dessas unidades. Diferente de valores cênicos, a análise dos serviços ambientais pode mais facilmente ser traduzida para termos econômicos (Ervin et al,. 2010; Groot et al., 2012; Gomez-Beggethun \& Martín-Lopez, 2015). Isso permite uma avaliação mais racional da efetividade das unidades de conservação e facilita o processo de tomada de decisão (Fuller et al. 2010, Saout et al. 2013), apesar de nem sempre haver relação ganha-ganha entre a manutenção de serviços ambientais e a conservação da natureza (Adams, 2014).

No Brasil, as unidades de conservação são regulamentadas pelo Sistema Nacional de Unidades de Conservação (SNUC), criado no ano 2000 pela lei 9.985. Mas os primeiros trabalhos que discutem a implantação e a gestão de tais áreas em termos econômicos datam já de 1999 (May et al., 2000). A partir desses estudos pioneiros consolida-se a visão de que a tradução da importância das unidades de conservação em termos monetários, estratégia chamada de valoração ambiental, pode ser ferramenta importante nos processos de implantação e de gestão dessas áreas. Essa abordagem é capaz de descrever de forma objetiva e mensurável, com base em valores de uso e de não-uso, o papel das unidades na 
manutenção de serviços ambientais essenciais (Motta, 1997; May et al., 2000; Silva, 2015). Com isso pode viabilizar uma alocação mais eficiente de recursos, inclusive possibilitando o pagamento pela utilização dos serviços prestados pelas unidades (Remoundou et al., 2009; Rietbergen-McCracken \& Abaza, 2013).

No entanto, apesar de uma década e meia nos separarem desses trabalhos pioneiros, a valoração ambiental ainda não é amplamente utilizada no país (Silva, 2015). Muitos dos problemas constatados à época ainda existem hoje, como a falta de justificativas claras para a criação de novas unidades de conservação e deficiências em relação ao financiamento necessário à sua manutenção (Fenker, 2013). Isso é preocupante, já que os próprios órgãos ambientais concordam que há problemas na gestão das unidades (IBAMA, 2007), e que, portanto, seriam necessárias novas metodologias para garantir a efetiva proteção de tais áreas.

Sobretudo no ambiente marinho, não se conhecem iniciativas que buscam quantificar a importância dos serviços ambientais prestados pelas unidades de conservação do país. Isso contrasta com ou tras regiões do planeta, onde a importância de abordagens econômicas na conservação da zona costeira tem sido ressaltada (Farrow, 1996; Chen et al., 2014; Brouwer et al., 2016; Trujillo et al., 2016). Devido à conectividade inerente aos ambientes marinhos, o funcionamento dessas unidades é por vezes bastante complexo e a utilização de critérios objetivos para descrever sua importância poderia contribuir sensivelmente na melhoria da sua gestão (MMA, 2010), sobretudo ao incorporar, nas políticas públicas, ações que minimizem as falhas de mercado decorrentes da natureza dos bens públicos e da existência de externalidades (Pindyck \& Rubinfeld, 2013)

O presente trabalho tem por objetivo estimar os valores de uso e de não uso do Parque Estadual Marinho da Pedra da Risca do Meio (PRM), única unidade de conservação exclusivamente marinha do estado do Ceará. Essa unidade de conservação protege ambientes peculiares da costa brasileira (Soares et al., 2016), porém, ainda carece de uma gestão eficiente, inclusive por conta da falta de dados que permitam tomadas de decisão bem informadas (Lima Filho, 2006, Soares et al., 2011). Portanto, mas com consequências que podem ir além de questões administrativas, é preciso que sejam realizados estudos básicos da região, dentre os quais se inclui a valoração. Evidências econômicas oriundas de estimativas mais claras dos valores de uso e não-uso dessa unidade poderiam dar suporte aos gestores nos processos de aquisição e alocação de recursos. Além disso, a estimativa desses valores pode servir de base de comparação para outras unidades de conservação, estimulando a adoção de estratégias de manejo mais adequadas, como ações de divulgação, controle da visitação, zoneamento e criação de zonas de amortecimento. Assim espera-se contribuir tanto para a otimização da gestão do PRM, quanto de outras unidades de conservação marinha do país.

\section{MATERIAL E MÉTODOS}

\section{Área de estudo}

O PRM, com uma área de 33,20 km², está localizado a 10 milhas náuticas (aproximadamente $18 \mathrm{~km}$ ) na direção $60^{\circ} \mathrm{NE}$ do município de Fortaleza, estado do Ceará (Figura 1). É a única unidade de conservação exclusivamente marinha do estado e foi criada para proteger recifes de arenito e bancos de sedimento, que abrigam rica biota (Soares et al., 2016). Essas feições estão permanentemente submersas, em profundidades que variam de 17 a $30 \mathrm{~m}$. 


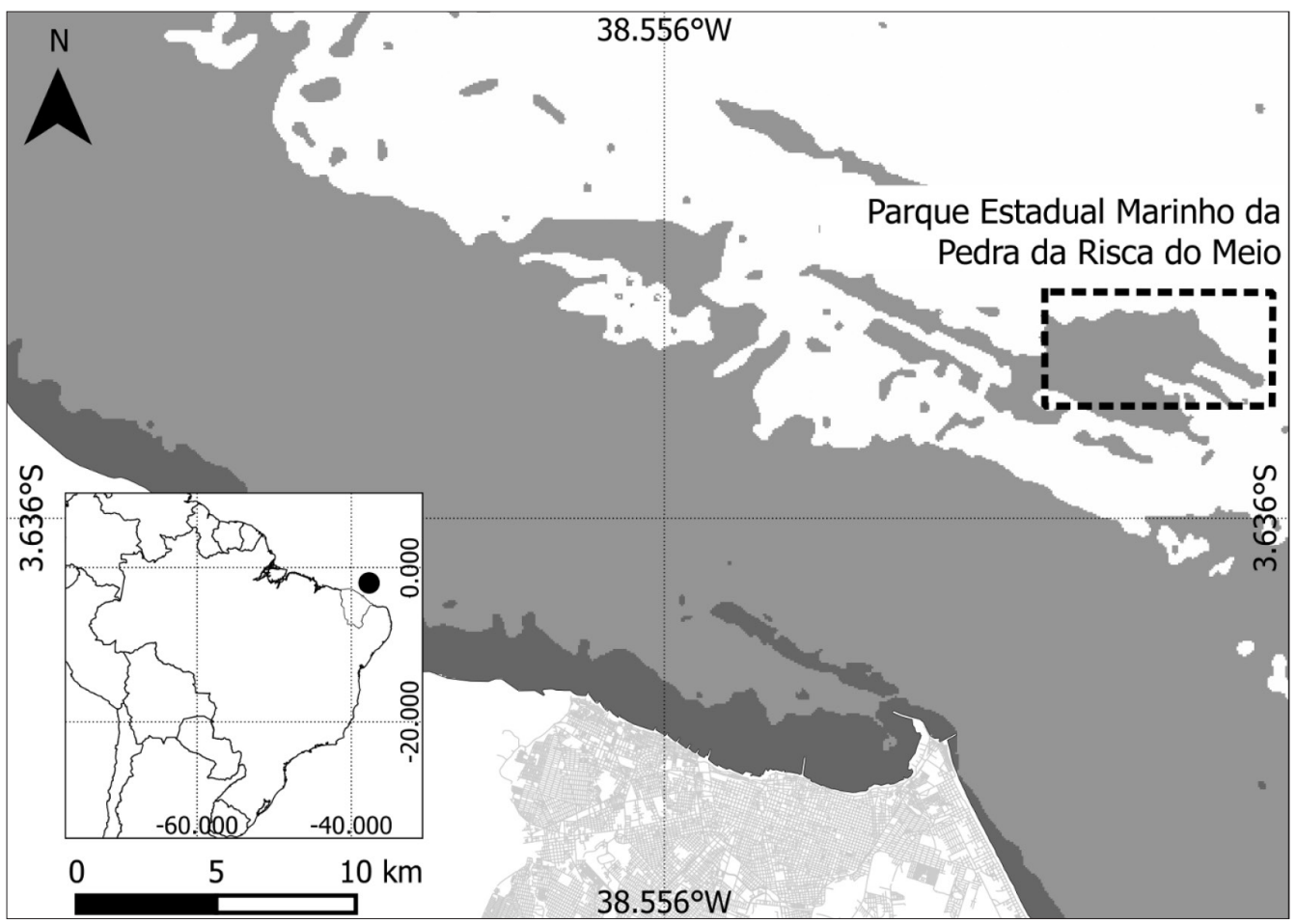

Figura 1 - Localização do Parque Estadual Marinho da Pedra da Risca do Meio em relação a costa da cidade de Fortaleza/Ceará.

O PRM foi criado pela Lei Estadual 12.717 de 05 de setembro de 1997 e já no seu artigo $1 .^{\circ}$ coloca como única finalidade a proteção integral à flora, à fauna e às belezas cênicas daquele ecossistema. Apesar de afirmar a proteção integral, esse dispositivo legal considerou os usos históricos da área do Parque e permitiu a pescaria artesanal com linha e anzol dentro da unidade de conservação, mesmo tendo proibido outros tipos de pesca. Essa permissão, no entanto, está em desacordo com a lei de criação do SNUC, que proíbe todo tipo de exploração econômica de Parques públicos, com exceção do turismo ecológico. Apesar de ainda não haver uma análise do assunto sob o ponto de vista legal, alguns autores concordam que a aparente ambiguidade entre os dispositivos estadual e federal que dificulta a gestão do Parque, por vezes impedindo a entrada de recursos para sua gestão (Soares et al., 2011).

Visualmente, a área do PRM é biologicamente muito rica. No entanto, são escassos os estudos sobre o local e praticamente inexistem caracterizações abrangentes do Parque. Tem-se o registro documentado de que a região é frequentada por 123 espécies de peixes ósseos, seis de peixes cartilaginosos, uma de golfinho e três de tartaruga, muitas dessas ameaçadas de extinção (Freitas, 2009; Soares et al., 2011). Também sabe-se que grande parte da superfície dos recifes está coberta por esponjas, corais e algas, sem no entanto termos a identificação da maioria das espécies (Soares et al., 2016). Informalmente, também são comuns os relatos da grande abundância de lagosta sobre os recifes do Parque. Apesar dos poucos estudos, é importante ressaltar que pelo menos uma espécie nova de esponja foi descrita com material encontrado no PRM (Salani et al., 2006) e que duas espécies novas de algas, ainda não formalmente descritas, foram encontradas apenas no Parque e em trechos do litoral do estado do Espírito Santo (Sissini, 2013), o que reforça tanto a importância da área quanto a de novos estudos sobre a mesma. 
Devido à elevada riqueza de espécies, à falta de fiscalização e às contradições legais, a unidade de conservação tem sido muito visada para usos que vão além até mesmo da pesca artesanal com linha e anzol. São comuns os relatos informais de pesca com apetrechos proibidos como compressores, redes ou armadilhas (marambaias). Esses usos, além de ilegais, têm sido danosos ao PRM, com mergulhadores reportando frequentemente a ocorrência de restos desses apetrechos perdidos, presos aos recifes, ameaçando de forma continuada a fauna residente (Andrade \& Soares, 2017).

\section{Valoração ambiental}

A técnica de valoração ambiental é uma ferramenta da Economia Neoclássica usada para valorar os bens e serviços para os quais não existe mercado. No presente, foi adotada a metodologia de valoração contingente (MVC), que consiste em medir, através de perguntas diretas, o quanto as pessoas estariam dispostas a pagar ou receber por determinado bem ou serviço ambiental (Motta, 2006). Esse método mensura monetariamente o impacto no bem-estar das pessoas proveniente de uma variação na qualidade dos bens ou serviços ambientais estudados. Tal metodologia tem a vantagem de estimar o valor econômico total das unidades de conservação, além dos seus valores de uso direto ou indireto (Loomis et al., 2000; Zhongmin et al., 2003). Isso ajuda a mitigar algumas das dúvidas que surgem quando se trata de pagamentos por serviços ambientais, mas por outro lado exige cuidados redobrados para garantir a consistência e a coerência dos dados (Arrow et al., 1993). Os valores encontrados por tal metodologia não são necessariamente cobrados, mas devem ser contemplados nos processos de tomada de decisão e na determinação de punição/ compensação por danos ambientais, evitando assim a exploração excessiva dos bens e serviços ambientais (Motta, 2006).

O valor econômico dos recursos ambientais deriva de atributos que podem ou não estar associados ao seu uso. Dessa forma, o valor econômico ambiental pode ser decomposto em valor de uso (i.e., decorrentes da utilização direta, normalmente com fins econômicos) e valor de não uso (i.e. valores externos ao mercado, como aqueles de fundo cultural e estético), sendo o método de valoração contingente o único capaz de determinar o valor de existência de um recurso natural (Motta, 2006). No caso do PRM temos o uso por parte dos mergulhadores e pescadores, e o valor de existência associado ao direito de existência de outras espécies e de riquezas naturais.

O MVC estima o valor da disposição a pagar (DAP) baseado em mercados hipotéticos simulados em pesquisas de campo por meio de questionários (ANEXO). No presente estudo, tais questionários foram elaborados considerando as recomendações do relatório da National Oceanic and Atmospheric Administration (NOAA) sobre o método de valoração contingente (Arrow et al., 1993), ou seja, com perguntas sobre a frequência de visitação e o conhecimento à respeito do PRM; com o uso de um veículo de pagamento que reflita uma situação real (uma quantia para manutenção e conservação do parque); com o uso de pesquisa piloto; com o uso da DAP; e, com o uso de fotografia sem impacto emocional.

Ao todo foram aplicados 107 questionários entre os meses de outubro e novembro de 2014. Desse total, dois foram considerados discrepantes por apresentarem valores de disposição a pagar consideravelmente mais elevadas do que a média. Assim todas as análises foram feitas considerando 105 entrevistas. Apesar do tamanho da amostra, esse número ainda é adequado do ponto de vista do poder do teste de inferência estatística, que se torna aceitável para tamanhos amostrais acima de 100 (Hair et al., 2005). 
Os questionários foram aplicados à moradores de Fortaleza, capital do estado do Ceará, e centro urbano mais próximo do PRM, visando medir a disposição desta população a pagar pela manutenção do PRM nas suas condições atuais. Em geral os entrevistados estavam relacionados direta ou indiretamente ao mar: pescadores, armadores de pesca, mergulhadores, estudantes, pesquisadores, professores, vendedores de pescado, etc. Além destes, foram entrevistados outros profissionais como engenheiros, analistas, economistas e psicólogos.

Ao todo, foram realizadas 74 entrevistas com pessoas que nunca tinham ido ao Parque e 31 com pessoas que já estiveram no local. O primeiro grupo foi utilizado na estimativa dos valores de não-uso, enquanto o segundo dos valores de uso (uma distinção frequente na literatura, por exemplo Pendleton et al., 2007; Wallmo \& Edwards, 2008; McVittie \& Moran, 2010). Ambos os valores foram calculados a partir da disposição à pagar média, manifestada espontaneamente pelos entrevistados. Vale ressaltar que, dentre os visitantes do Parque, foram entrevistadas pessoas que foram ao PRM para lazer e/ou a trabalho.

A aplicação dos questionários foi precedida pela apresentação de fotografias do PRM selecionadas dentre imagens disponíveis na internet. Essa etapa foi utilizada para reduzir a variância ao se comparar pessoas que já mergulharam no Parque, e que, portanto, têm maior conhecimento do mesmo, com pessoas que utilizam a unidade de conservação para a pesca ou que nunca ouviram falar desta.

Para a realização das entrevistas, foram feitas algumas adaptações das recomendações da NOAA (Arrow et al., 1993). Esse relatório recomenda a realização de entrevistas presenciais, evitando-se entrevistas por telefone ou correio. No entanto, levando em consideração os avanços tecnológicos dos últimos 20 anos que aumentaram nossa capacidade de comunicação à distância, e seguindo o exemplo de outros autores (ex. Kramer et al., 1995; Santos-Corrêa, 2005; Lindhjem \& Navrud, 2011; Camargo, 2015), foram realizadas 64 entrevistas via internet, além de 41 entrevistas presenciais. No caso das entrevistas por internet, as fotos foram encaminhadas precedendo os questionários, permitindo uma pronta e fácil visualização das mesmas.

Outra recomendação diz respeito a realização de teste prévio das fotografias a serem apresentadas aos entrevistados para introdução da área a ser valorada. Virtualmente todas as fotos do PEMPRM foram tiradas por mergulhadores enfocando suas próprias atividades ou a vida marinha. Portanto, selecionamos imagens que correspondessem a esses temas, mas, devido à homogeneidade de assuntos nas fotografias, não foi feito o pré-teste.

Por fim, o relatório da NOAA recomenda que os questionários sigam o formato de referendo, quando é proposto um valor e os entrevistados respondem apenas se aceitam ou não pagá-lo. No entanto, levando em consideração o exposto por Green et al., (1998), o presente estudo usou questionários com perguntas abertas que produzem uma variável contínua de lances. Nesse caso, valor da disposição a pagar pode ser estimado diretamente por técnicas econométricas (Motta, 2006).

\section{Análises estatísticas}

A disposição a pagar (DAP) e o perfil socioeconômico dos entrevistados foram analisados por meio de estatísticas descritivas. Para estimar valores de uso (i.e. utilização direta, indireta e de opção) e de não uso (i.e. valor de existência, externo ao mercado), foram comparados os valores da DAP de entrevistados que já foram ao Parque (usuários) 
e de entrevistados que nunca foram ao Parque (não usuários). Assim, a DAP manifestada por não usuários foi considerada como uma medida do valor de não uso, enquanto que a diferença entre essa e a DAP dos usuários foi entendida como uma medida do valor de uso. Além disso, foi feita uma comparação entre os dois grupos que, por considerar a natureza enviesada e não-normal dos dados (devido ao número de valores nulos), foi feita via teste U de Mann-Whitney. Considerando a diferença no número de entrevistados usuários e não usuários ( $n=31$ e 74, respectivamente), o que diminui a potência do teste e aumenta a chance de erro tipo II (Fagerland \& Sandvik, 2009), decidiu-se adotar um nível de significância menos rigoroso nessa análise. Assim o teste foi considerado significativo se valor-p < 0,10 (Gujarati, 2006; Larson \& Farber, 2010; Zar, 2010).

Para entender quais fatores contribuíram para o comportamento da DAP foram construídos modelos de regressão linear múltipla, utilizando como variáveis o gênero, a idade, o grau de instrução, a renda, o conhecimento prévio do parque e a visitação prévia ao Parque, informações essas que foram obtidas durante as entrevistas. Para a seleção do modelo mais adequado foi utilizado o método backward. Esse método consiste na geração sucessiva de funções lineares, cada uma com uma variável explicativa a menos (Fávero et al., 2009). A função mais adequada para a representação dos dados deve apresentar maior poder explicativo (maior $\mathrm{R}^{2}$ ) e menor quadrado médio do erro, também considerando-se significativo o modelo com valor-p $<0,10$.

Por fim, a disposição a pagar total (DAPT) de usuários e não usuários foi calculada multiplicando a DAP média em cada faixa de renda, pela proporção de entrevistados incluídos naquela faixa, e pela população total de usuários e não usuários do Parque em Fortaleza, seguindo a fórmula de Motta (1997).

Para tanto, a população total de usuários foi definida como o número de mergulhadores cadastrados e de pescadores ativos no município. Somando-se os dados obtidos junto às duas maiores escolas de mergulho da cidade (Atlântida Serviços Marítimos e Mar do Ceará, comunicação pessoal) existem cerca de 3.000 mergulhadores em Fortaleza. Esse é o mesmo número de pescadores ativos segundo a colônia de pescadores do município (Colônia Z8, comunicação pessoal), o que o totaliza aproximadamente 6.000 usuários.

Já a população dos não usuários, pelo perfil socioeconômico dos entrevistados, foi considerada como sendo a fração da população de Fortaleza com renda per capta superior a $\mathrm{R} \$ 914,00$ (renda familiar superior a $\mathrm{R} \$ 3.656,00$ ). Isso equivale a 491.920 pessoas no ano de 2010 (Menezes \& Medeiros, 2012).

\section{RESULTADOS}

\section{Perfil dos entrevistados}

O perfil dos entrevistados, divididos entre os que já foram $(n=31)$ e os que ainda não foram ( $\mathrm{n}=74)$ ao PRM, são apresentados na Tabela I. A maioria dos entrevistados foram homens, entre 21 e 40 anos, com elevado grau de escolaridade e renda mensal entre $\mathrm{R} \$ 1.501,00$ e $\mathrm{R} \$ 5.000,00$. Cabe ressaltar que, apesar de a maioria dos usuários entrevistados serem mergulhadores (Figura 2), atividade essa que normalmente demanda um maior poder aquisitivo, a proporção de usuários e não usuários acabou sendo maior na faixa de renda entre $\mathrm{R}$ \$ 0,00 e R \$ 750,00 e menor grau de instrução. 
Tabela I - Características socioeconômicas dos entrevistados.

\begin{tabular}{|c|c|c|c|}
\hline & $\begin{array}{c}\text { Foi ao } \\
\text { Parque }\end{array}$ & $\begin{array}{l}\text { Não foi ao } \\
\text { Parque }\end{array}$ & TOTAL \\
\hline \multicolumn{4}{|l|}{ Sexo } \\
\hline Feminino & 9 & 37 & 46 \\
\hline Masculino & 22 & 37 & 59 \\
\hline \multicolumn{4}{|l|}{ Renda } \\
\hline $\mathrm{R} \$ 0,00$ - $\mathrm{R} \$ 750,00$ & 11 & 12 & 23 \\
\hline $\mathrm{R} \$ 751,00$ - R\$ 1500,00 & 5 & 14 & 19 \\
\hline $\mathrm{R} \$ 1501,00-\mathrm{R} \$ 5000,00$ & 9 & 36 & 45 \\
\hline Acima de $\mathrm{R} \$ 5000,00$ & 6 & 12 & 18 \\
\hline \multicolumn{4}{|l|}{ Idade } \\
\hline$\geq 18$ & 0 & 4 & 4 \\
\hline 21 à 30 & 7 & 44 & 51 \\
\hline 31 à 40 & 10 & 15 & 25 \\
\hline 41 à 50 & 4 & 4 & 8 \\
\hline 51 à 60 & 6 & 5 & 11 \\
\hline$>60$ & 4 & 2 & 6 \\
\hline \multicolumn{4}{|l|}{ Instrução } \\
\hline Ensino fundamental incomplete & 9 & 2 & 11 \\
\hline Ensino fundamental completo & 0 & 0 & 0 \\
\hline Ensino médio incompleto & 3 & 1 & 4 \\
\hline Ensino médio completo & 2 & 5 & 7 \\
\hline Graduação incompleta & 2 & 10 & 12 \\
\hline Graduação completa & 4 & 33 & 37 \\
\hline Pós-graduação & 11 & 23 & 34 \\
\hline
\end{tabular}

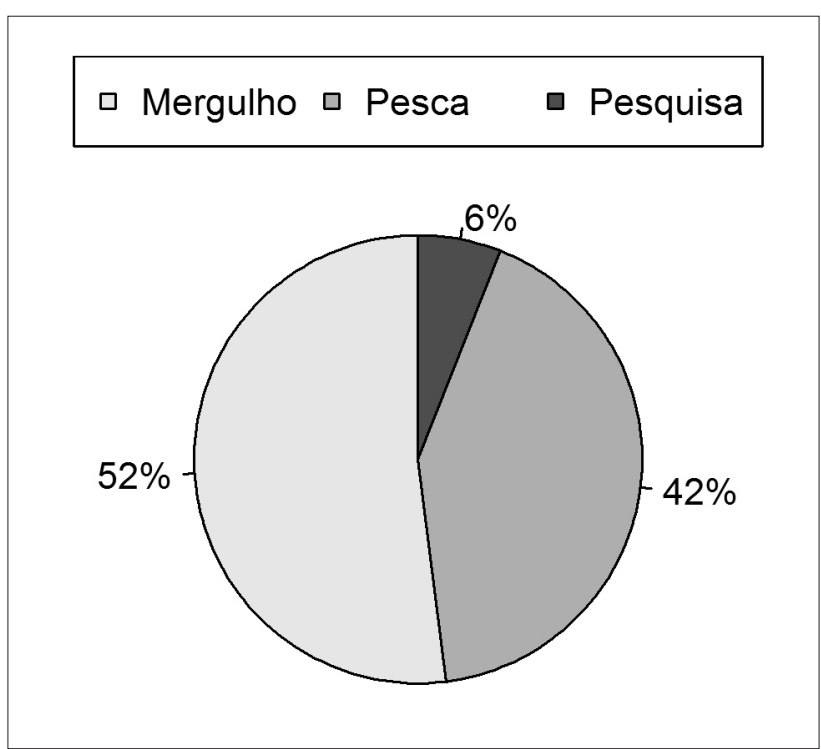

Figura 2 - Usos do Parque Estadual Marinho da Pedra da Risca do Meio pelos visitantes entrevistados no presente estudo.

\section{Disposição a Pagar}

A disposição a pagar (DAP) mensal pela manutenção do Parque Estadual Marinho da Pedra da Risca do Meio variou de $\mathrm{R} \$ 0,00$ a $\mathrm{R} \$ 200,00$ com média de $\mathrm{R} \$ 21,50 \pm 34,28$. No entanto grande parte dos entrevistados $(41,90 \%)$ não estiveram dispostos a pagar pela manutenção do Parque, alegando que essa é uma despesa de responsabilidade do Estado 
(Figura 3). Com isso, a distribuição de frequência da DAP ficou fortemente enviesada para a esquerda (Figura 4A). Por conta dessa distribuição não normal, a mediana poderia representar os dados mais adequadamente que a média. Esse valor ficou em R \$10,00 e, baseado no critério dos quartis, qualquer valor acima de $\mathrm{R} \$ 100,00$ pode ser considerado um outlier (Figura 4B). Vale ressaltar que, mesmo removendo os resultados nulos, mais da metade $(54,08 \%)$ dos entrevistados pagariam menos de $\mathrm{R} \$ 50,00$ por mês.

Discriminando os resultados da DAP, uma proporção maior de usuários se mostrou disposta a pagar por mês pela manutenção do Parque quando em comparação com os não usuários (74\% e 51\%, respectivamente). Além disso, o valor indicado pelos usuários foi significativamente maior $(\mathrm{U}=887, \mathrm{p}=0,07)$, tendo sido de $\mathrm{R} \$ 30,94 \pm 44,39$ contra $\mathrm{R} \$ 17,55$ $\pm 28,47$. Representando esses dados como mediana, que presenta mais facilmente o resultado do teste estatístico, esses valores seriam respectivamente de $\mathrm{R} \$ 10,00$ e $\mathrm{R} \$ 3,00$.

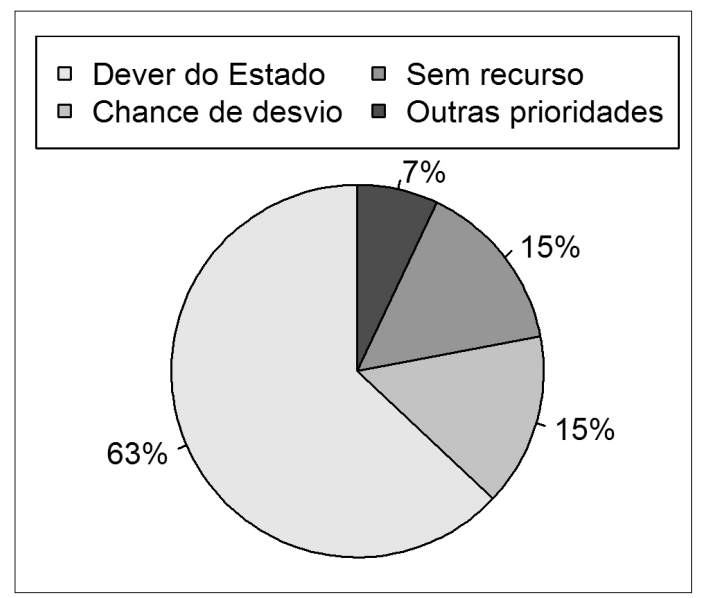

Figura 3 - Principais justificativas apontadas por quem não está disposto a pagar pela preservação do Parque.

\section{Modelo de Disposição a Pagar}

O método backward para a seleção das variáveis explicativas da DAP mostrou que o modelo com maior poder explicativo $\left(\mathrm{R}^{2}=0,32\right)$ foi aquele que incluiu todas as variávies analisadas, com excessão de conhecimento do Parque (Tabela II). Esse modelo foi estatisticamente significativo $(\mathrm{p}$ $<0,1$ ) e pode ser expresso como:

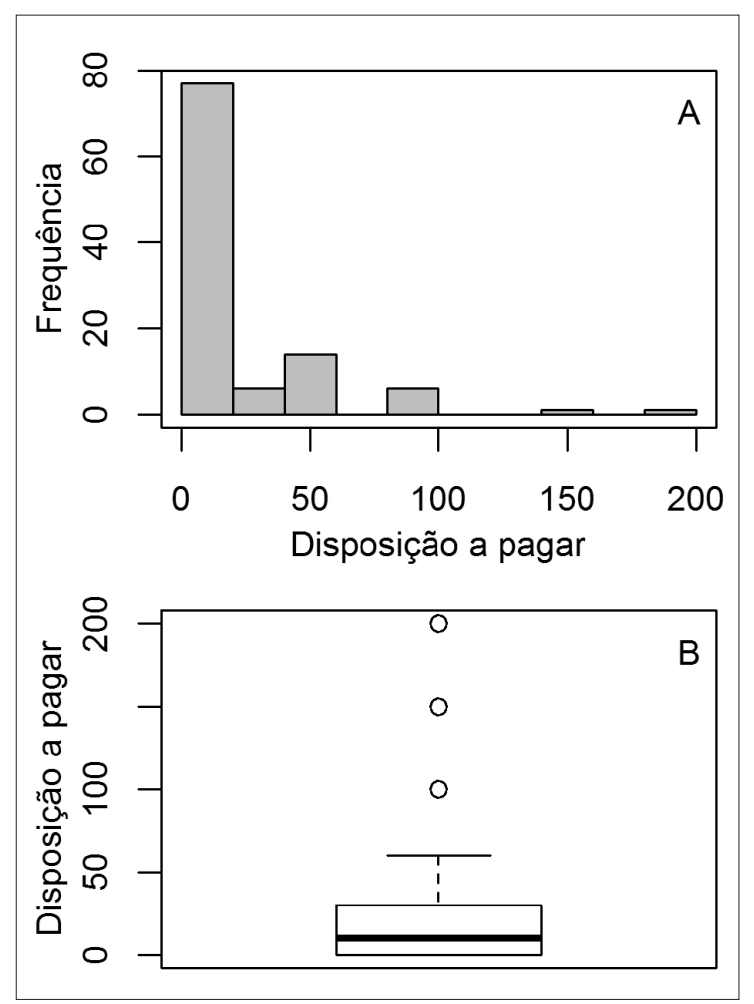

Figura 4 - Distribuição de frequências da disposição a pagar (DAP) por mês para a manutenção do Parque Estadual Marinho da Pedra da Risca do Meio - CE. A - Histograma mostrando a distribuição enviesada da DAP; B - Boxplot mostrando mediana, quartis e outliers da DAP.

$D A P=-6,154+10,558 G_{i}+0,061 I_{i}+9,146 R_{i}+15,132 V_{i}+4,305 S_{i}-11,351 U_{i}$

Onde:

$D A P=$ Disposição a pagar

$G_{i}=$ Gênero dos indivíduos (1 - feminino, 0 - masculino)

$I_{i}=$ Idade dos indivíduos em anos

$R_{i}=$ Renda mensal dos indivíduos em número de salários mínimos

$V_{i}=$ Se os indivíduos visitaram ou não o Parque previamente (1 - sim, 0 - não) 
$S_{i}=$ Segundo grau

$U_{i}=$ Superior completo

Dentre as variáveis estudadas, apenas renda e visitação prévia tiveram influência significativa na DAP. Em outras palavras, quanto maior a renda dos entrevistados e a possibilidade de efetiva utilização do Parque, maior a disposição a pagar pela conservação do mesmo.

\section{Disposição a Pagar Total}

Os dados de disposição a pagar total (DAPT) são apresentados na Tabela III. Considerando a DAP dos não usuários como uma estimativa do valor de não uso, é possível calcular, pela diferença entre as $\mathrm{DAP}^{\prime}$ s de usuários e não usuários, um valor de $\mathrm{R} \$ 13,39$ por pessoa por mês, que seria representativo do valor de uso do Parque. Levando em conta a população de usuários, esse valor de uso equivale a uma DAPT de $\mathrm{R} \$ 80.340,00$ mensais, ou R\$ 964.080 por ano. É importante ressaltar que esse montante se refere apenas ao valor dos serviços efetivamente realizados no e pelo Parque, e desconsidera, por exemplo seus valores estéticos e culturais.

Tabela III - Classes de intervalo, valores médios da disposição a pagar (DAP) e disposição a pagar total (DAPT) pela manutenção do Parque Estadual Marinho da Pedra da Risca do Meio entre usuários e não usuários. $\mathrm{DAPM}=$ disposição a pagar média. $\mathrm{n}=$ número de pessoas por faixa de renda. $\mathrm{N}=$ número total de entrevistas. População = tamanho estimado da população de frequentadores $(n=6.000)$ e não frequentadores $(n=491.920)$.

\begin{tabular}{|c|c|c|c|c|}
\hline Faixa de renda & DAPM & $\mathbf{n}$ & $\begin{array}{c}\% \\
(\mathrm{n} / \mathrm{N})\end{array}$ & $\begin{array}{l}\text { Valor total do Parque } \\
\text { (DAPM x [n/N] x } \\
\text { população) }\end{array}$ \\
\hline \multicolumn{5}{|c|}{ NÃO FREQUENTADORES } \\
\hline $\mathrm{R} \$ 0,00$ & 0,00 & 36 & 0,49 & 0.00 \\
\hline $\mathrm{R} \$ 0,01$ a $\mathrm{R} \$ 10,00$ & 8,00 & 9 & 0,12 & $478.624,86$ \\
\hline $\mathrm{R} \$ 10,01$ a $\mathrm{R} \$ 20,00$ & 18,75 & 12 & 0,16 & $1.495 .702,70$ \\
\hline $\mathrm{R} \$ 20,01$ a $\mathrm{R} \$ 50,00$ & 42,31 & 13 & 0,18 & $3.656 .361,59$ \\
\hline $\mathrm{R} \$ 50,01$ a $\mathrm{R} \$ 100,00$ & 100,00 & 3 & 0,04 & $1.994 .270,27$ \\
\hline$>\mathrm{R} \$ 100,00$ & 150,00 & 1 & 0,01 & 997.135,14 \\
\hline TOTAL & & 74 & 1,00 & $8.622 .094,56$ \\
\hline \multicolumn{5}{|l|}{ FREQUENTADORES } \\
\hline $\mathrm{R} \$ 0,00$ & 0,00 & 8 & 0,26 & 0.00 \\
\hline $\mathrm{R} \$ 0,01$ a $\mathrm{R} \$ 10,00$ & 5,50 & 8 & 0,26 & $8.516,13$ \\
\hline $\mathrm{R} \$ 10,01$ a $\mathrm{R} \$ 20,00$ & 18,75 & 4 & 0,13 & $14.516,13$ \\
\hline $\mathrm{R} \$ 20,01$ a $\mathrm{R} \$ 50,00$ & 46,67 & 6 & 0,19 & $54.193,55$ \\
\hline $\mathrm{R} \$ 50,01$ a $\mathrm{R} \$ 100,00$ & 90,00 & 4 & 0,13 & $69.677,42$ \\
\hline$>\mathrm{R} \$ 100,00$ & 200,00 & 1 & 0,03 & $38.709,68$ \\
\hline TOTAL & & 31 & 1,00 & $185.612,90$ \\
\hline
\end{tabular}

- Backward para seleção de modelos preditivos da disposição Risca do Meio.

\begin{tabular}{lcccc}
\hline Variáveis explicativas & $\begin{array}{c}\text { Coeficiente } \\
\text { de Regressão }\end{array}$ & $\begin{array}{c}\text { Erro- } \\
\text { padrão }\end{array}$ & Teste-t & Valor-p \\
\hline Gênero & 10,558 & 7,196 & 1,467 & 0,146 \\
Idade & 0,061 & 0,407 & 0,150 & 0,881 \\
Renda & $9,146^{*}$ & 4,487 & 2,038 & 0,044 \\
Visitação prévia & $15,132^{*}$ & 8,097 & 1,869 & 0,065 \\
Segundo Grau** & 4,305 & 18,144 & 0,237 & 0,813 \\
Superior Completo** & $-11,351$ & 18,811 & $-0,603$ & 0,548 \\
Constante & $-6,154$ & 23,153 & $-0,266$ & 0,791 \\
Teste F & 1,857 & & & 0,096 \\
\hline
\end{tabular}

* denota a significância estatística ao nível de 10\%.

**Em caso de variável categórica o complemento é omitido. O complemento do grau de instrução é Analfabeto à Segundo Grau Incompleto. 


\section{DISCUSSÃO}

A valoração ambiental possui um grande potencial para auxiliar na tomada de decisão em ações de conservação, sobretudo pela possibilidade de incluir evidências econômicas em uma análise que muitas vezes é puramente ecológica (RietbergenMcCracken \& Abaza, 2000; Remoundou et al., 2009; Börger et al., 2014). No entanto, essa estratégia ainda não é amplamente adotada no Brasil, nem em análises científicas, muito menos pelos órgãos ambientais responsáveis para elaboração e execução das políticas ambientais (Silva, 2015). Pelo nosso conhecimento, este talvez tenha sido o primeiro estudo a expressar a importância de uma unidade de conservação marinha brasileira através de valores monetários.

Dentre as diferentes metodologias utilizadas para calcular o valor de bens e serviços ambientais, a valoração contingente possui a vantagem de poder estimar valores de não uso, externos ao mercado (Loomis et al., 2000; Zhongmin et al., 2003). Com isso, ela pode fornecer uma visão mais geral da importância desses ambientes, sendo particularmente útil em estudos iniciais (ex. Brouwer et al., 2016; Trujillo et al., 2016). No entanto, os resultados obtidos refletem a situação socioeconômica e o grau de conscientização da população em determinado momento, além de poderem variar a depender da metodologia utilizada (Arrow et al., 1993). Assim, as estimativas obtidas no presente estudo não podem ser diretamente generalizadas ou comparados com o de outras áreas, ou mesmo de outros momentos econômicos (Martinez-Alier et al., 1998). Portanto, são necessárias novas pesquisas para refinar as análises aqui apresentadas, principalmente incluindo uma quantificação explícita dos serviços ambientais prestados pelo PRM (Seppelt et al., 2011), adotando assim uma abordagem mais detalhada, que vem sendo recomendada por outros autores (Beaumont et al., 2008; Luisetti et al., 2011).

Além disso, é preciso reconhecer que a importância dos bens e serviços ambientais possui uma natureza múltipla (Farber et al., 2002; Groot et al., 2012). E nem sempre um índice (como o valor monetário produzido pelos métotos de valoração) é capaz de refletir com facilidade todos esses aspectos (Gomez-Beggethun \& Martín-Lopez, 2015). Portanto, a gestão do Parque não pode se restringir à consideração dos valores ora apresentados, sendo sempre necessária a consideração de fatores ecológicos e culturais para se conseguir um grau suficiente e sustentável de conservação.

Apesar dessas limitações, o método de valoração se mostrou capaz de fornecer dados objetivos que podem auxiliar a conservação do Parque. Por exemplo, nossos resultados concordam com estudos que apontam que o grau de informação é essencial no reconhecimento da importância de bens e serviços ambientais (Kollmuss \& Agyeman, 2002). Mais ainda, mostram que um contato direto com as Unidades de Conservação, através de experiências prévias, enseja uma percepção significativamente maior do valor das mesmas (Chawla, 1998). Dessa forma, sugere-se que ações de divulgação e o incentivo à visitação, quando em conjunto com estratégias de manejo adequadas, são potencialmente úteis na viabilização de unidades de conservação marinhas, tanto do ponto de vista político quanto financeiro. Inclusive por incentivar uma maior participação da população em geral e da sociedade civil organizada na gestão desses espaços, contribuindo assim para sua governança.

Especificamente em relação ao PRM, seu valor de uso equivale à quase $\mathrm{R} \$ 1.000 .000,00$ anuais. Esse valor mostra claramente a elevada importância dessa unidade de conservação. Considerando que esse Parque é relativamente pouco conhecido, existe um claro potencial 
de aumento no número de visitantes, o que, dada a DAP registrada no presente estudo (média de R\$21,50 por mês), pode ser convertido em uma fonte extra de recursos (ReidGrand \& Bhat, 2009; Thur, 2010). Isso fica ainda mais evidente quando considera-se que todas aos entrevistados manifestaram interesse na conservação do Parque, e que a maioria (58\%) se mostrou disposta a pagar por isso, um percentual que foi ainda maior entre os usuários (74\%). Por outro lado, entre os não-pagantes, a maioria alegou que não pagaria por isso ser atribuição do Estado, o que pode ser interpretado como um aval dos contribuintes para uma maior alocação de recursos na área. Portanto, uma estratégia de financiamento mista, que envolva tanto impostos quanto a cobrança de taxas dos frequentadores, parece, a priori, ser uma forma de alocação de recursos bem aceita pela população local.

Vale ressaltar que, apesar de os valores exatos não poderem ser generalizados, as questões aqui levantadas, tanto em relação à importância quanto ao financiamento do PRM, podem se aplicar à outras unidades de conservação marinhas similares. Por exemplo, no Brasil os parques marinhos do Parcel de Manuel Luís, de Fernando de Noronha e dos Abrolhos são conhecidos pelas elevadas biodiversidade e beleza cênica, o que atrai turistas, mergulhadores e pescadores, apesar de a atuação dos últimos ser ilegal (Vitali, 2009; Dutra et al., 2011; Piedade, 2013). Assim, é de se esperar que essas Unidades também tenham valores de uso e de não uso bastante elevados. Se por um laod isso pode viabilizar a adoção de estratégias de cobrança pela sua conservação (algo que já ocorre, por exemplo, em Fernando de Noronha e Abrolhos), por outro sugere que esses Parques estão ou podem vir a estar sob intensa exploração econômica. Essa exploração pode trazer grandes prejuízos à essas áreas (Silva Jr., 2003; Feitosa \& Gomez, 2013), e para garantir a manutenção dos seus valores de não uso, deveria ser bem controlada, o que demanda novas e criativas estratégias de financiamento para garantir sua conservação.

Por fim, os resultados desse estudo indicam que, além de uma utilização consolidada e relevante dos serviços gerados pelo PRM, essa Unidade também exibe um elevado valor de não uso. Assim, na discussão sobre a readequação legal da sua categoria de proteção, ambos os valores de uso e de não uso precisam ser levados em consideração para garantir a efetiva conservação dos bens e serviços abrangidos pelo PRM. Portanto, a melhor estratégia parece ser adotar uma categoria de proteção integral, que efetivamente preserve os ecossistemas dentro do Parque, mas que ao mesmo tempo não restrinja excessivamente o acesso à área, garantindo assim a manutenção de ambos os valores. Nesse caso, a atual categoria de Parque parece ser de fato a mais adequada, porém é necessário que os gestores sejam capazes de coibir a pesca no polígono da unidade de conservação, incentivando, por exemplo, o uso de áreas adjacentes (Rassweiler et al., 2012).

A abordagem da valoração ambiental se mostrou útil no levantamento de evidências econômicas da importância do Parque Estadual Marinho da Pedra da Risca do Meio, apontando alguns possíveis caminhos para a gestão financeira dessa Unidade, como por exemplo a existência de apoio público ao investimento estatal e a possibilidade de cobrança de taxas dos frequentadores. E, como estudo de caso, nossos resultados podem ajudar na gestão de outras áreas ao longo da zona costeira do Brasil. Novas pesquisas, incluindo um levantamento mais explícito do papel ecológico das Unidades de Conservação marinhas, poderão refinar a presente análise, fornecendo uma visão mais concreta da importância das mesmas, e possibilitando a adoção de estratégias de financiamento inéditas no Brasil, como o pagamento pelos serviços ambientais prestados. 


\section{REFERÊNCIAS BIBLIOGRÁFICAS}

Adams, W.M. The value of valuing nature. Science, v. 346, n. 6209, p. 549-551, 2014.

Andrade, A. B. \& Soares, M. O. Offshore marine protected areas: divergent perceptions of divers and artisanal fishers. Mar. Policy v. 76, p. 107-113, 2017.

Arrow, K.; Solow, R.; Portney, P.R.; Leamer, E.E.; Radner, R. \& Schuman, H. Report of the NOAA panel on Contingent Valuation. Federal Register, v. 58, n. 10, p. 4601-4614, 1993.

Beaumont, N.J.; Austen, M.C.; Mangi, S.C. \& Townsend, M. Economic valuation for the conservation of marine biodiversity. Mar. Pollut. Bull., v. 56, n. 3, p. 386-396, 2008.

Börger, T.; Beaumont, N.J.; Pendleton, L.; Boyle, K.J.; Cooper, P.; Fletcher, S.; Haab, T.; Hanemann, M.; Hooper, T.L.; Hussain, S.S.; Portela, R.; Stithou, M.; Stockill, J.; Taylor, T. \& Austen, M.C. Incorporating ecosystem services in marine planning: The role of valuation. Mar. Policy, v. 46, p. 161-170, 2014.

Brouwer, R.; Brouwer, S.; Eleveld, M.A.; Verbraak, M.; Wagtendonk, A.J. \& van der Woerd, H.J. Public willingness to pay for alternative management regimes of remote marine protected areas in the North Sea. Mar. Policy, v. 68, p. 195-204, 2016.

Camargo, A.R. Áreas de lazer urbanas: um estudo de valoração econômica ambiental realizado no município de Londrina. Monografia de Graduação, Curso de Engenharia Ambiental, Universidade Tecnológica Federal do Paraná, 60 p. Londrina, 2015.

Chawla, J. Significant life experiences revisited: A review of research on sources of environmental sensitivity. J Environ Educ., v. 29, n. 3, p. 11-21, 1998.

Chen, J.L.; Chen, J.Y.; Chuang, C.T.; Lu, H.J.; Liu, H.H. \& Lin, Y.S. Developing a comanagement financing mechanism to enhance the financial sustainability of marine protected areas in Taiwan. Mar.Policy, v. 48, p. 126-133, 2014.

Dutra, G.G.; Camargo, E.; Santos, C.A.P. \& Ceotto, P. Abrolhos: desafios para a conservação e o desenvolvimento sustentável na área com a maior biodiversidade marinha do Atlântico Sul. Field Actions Science Reports, volume especial 3, 2011.

Ervin, J.; Sekhran, N.; Dinu, A.; Gidda, S.; Vergeichik, M. \& Mee, J. Protected Areas for the 21 st Century: Lessons from UNDP / GEF's Portfolio. United Nations Development Programme, 131 p., Nova York, 2010.

Fagerland, M. W. \& Sandvik, L. The Wilcoxon-Mann-Whitney test under scrutiny. Statistics in Medicine, v. 28, p. 1487-1497, 2009.

Farber, S. C.; Costanza, R. \& Wilson, M. A. Economic and ecological concepts for valuing ecosystem services. Ecol. Econ., v. 41, n. 3, p. 375-392, 2002.

Farrow, S. Marine protected areas: emerging economics. Mar. Policy v. 20, n. 6, p. 439446, 1996.

Fávero, L.P.; Belfiore, P.; Silva, F.L. \& Chan, B.L. Análise de dados: modelagem multivariada para tomada de decisões. Elsevier, 544 p., Rio de Janeiro, 2009.

Feitosa, M. J. S. \& Gomez, C. R. P. Aplicação do tourism ecological footprint method para avaliação dos impactos ambientais do turismo em ilhas: um estudo em Fernando de Noronha. Rev. Bras. Pesq. Tur., São Paulo, v. 7, n. 2, p. 220-238, 2013. 
Fenker, E.A. A valoração econômica dos recursos naturais na criação de unidades de conservação federais (UCF) no Brasil: Um estudo empírico no estado de Santa Catarina. Tese de Doutorado, Programa de Pós-graduação em Administração, Universidade do Vale do Itajaí, 304 p., Biguaçu, 2013.

Freitas, J.E.P. Ictiofauna do Parque Estadual Marinho da Pedra da Risca do Meio (Ceará - Brasil): composição, estrutua e contexto biogeográfico. Dissertação de Mestrado, Programa de Pósgraduação em Ciências Marinhas Tropicais, Universidade Federal do Ceará, 117 p., Fortaleza, 2009.

Fuller, R.A.; McDonald-Madden, E.; Wilson, K.A.; Carwardine, J.; Grantham, H.S.; Watson, J.E.M.; Klein, C.J.; Green, D.C. \& Possingham. H.P. Replacing underperforming protected areas achieves better conservation outcomes. Nature, v. 466, n. 7304, p. 365-367, 2010

Gómez-Baggethun, E. \& Martín-López, B. Ecological economics perspectives on ecosystem services valuation, 260-282, in Martínez-Alier, J. \& Muradian, R. (eds.) Handbook of Ecological Economics. Edward Elgar, 503 p. 2015.

Green, D.; Jacowitz, K.E.; Kahneman, D. \& McFadden, D. Referendum contingent valuation, anchoring, and willingness to pay for public goods. Resour. Energy. Econ., v. 20, n. 2, p. 85-116, 1998.

Groot, R.; Brander, L.; Ploeg, S.; Costanza, R.; Bernard, F.; Braat, L.; Christie, M.; Crossman, N.; Ghermandi, A.; Hein, L.; Hussain, S.; Kumar, P.; McVittie, A.; Portela, R.; Rodriguez, L.; ten Brink, P. \& van Beukering, P. Global estimates of the value of ecosystems and their services in monetary units. Ecosystem Services, v. 1, n. 1, p. 50-61, 2012.

Gujarati, D. Econometria Básica. 4.ed. Campus/Elsevier, Rio de Janeiro, 2006.

Hair, J.F.; Anderson, R.E.; Tatham, R.L. \& Black, W.C. Análise multivariada de dados. Bookman, 688 p., Porto Alegre, 2005.

IBAMA. Efetividade de gestão das unidades de conservação federais do Brasil. IBAMA, 96 p., Brasilia, 2007.

Kollmuss, A. \& Agyeman, J. Mind the gap: why do people act environmentally and what are the barriers to pro-environmental behavior? Environ. Educ. Res., v. 8, n. 3, p. 239-260, 2002.

Kramer, R. A.; Sharma, N.; Munasinghe, M. Valuing tropical forests: methodology and case study madagascar. The Word Bank, 66 p., Washington, 1995.

Larson, R; Farber, B. Estatística aplicada. 4. ed. Pearson Prentice Hall, São Paulo, 2010.

Lima Filho, J.F. Análise da efetividade de manejo de áreas marinhas protegidas: um estudo do Parque Estadual Marinho da Pedra da Risca do Meio. Dissertação de Mestrado, Programa de Pós-Graduação em Desenvolvimento e Meio Ambiente, Universidade Federal do Ceará, 134 p., Fortaleza, 2006.

Lindhjem, H.; Navrud, S. Using internet in stated preference surveys: a review and comparison of survey modes. International Review of Environmental and Resources Economics, v. 5, p. 309-351, 2011.

Loomis, J.; Kent, P.; Strange, L.; Fausch, K. \& Covich, A. Measuring the total economic value of restoring ecosystem services in an impaired river basin: results from a contingent valuation survey. Ecol. Econ., v. 33, n. 1, p. 103-117, 2000. 
Luisetti, T.; Turner, R.K.; Bateman, I.J.; Morse-Jonesa, S.; Adams, C. \& Fonseca, L. Coastal and marine ecosystem services valuation for policy and management: Managed realignment case studies in England. Ocean Coast. Manag., v. 54, n. 3, p. 212-224, 2011.

Martinez-Alier, J.; Munda, G. \& O'Neill, J. Weak Comparability of values as a foundation for ecological economics. Ecol. Econ, v. 26, n. 3, p. 277-286, 1998.

May, P.H.; Neto, F.C.V. \& Pozo, O.V.C. Valoração Econômica da biodiversidade. MMA, 200 p., Brasilia, 2000.

McVittie, A. \& Moran, D. Valuing the non-use benefits of marine conservation zones: an application to the uk marine bill. Ecol. Econ., v. 70, n. 2, p. 413-424, 2010.

Menezes, A.S.B. \& Medeiros, C.N. Perfil socioeconômico de Fortaleza. IPECE, 186 p., Fortaleza, 2012.

MMA. Panorama da conservação dos ecossistemas costeiros e marinhos no Brasil. MMA, 148 p., Brasilia, 2010.

Motta, R.S. Economia ambiental. Editora FGV, 228 p., Rio de Janeiro, 2006.

Motta, R.S. Manual para valoração econômica de recursos ambientais. IPEA/MMA, 242 p., Rio de Janeiro, 1997.

Pendleton, L.; Atiyah, P. \& Moorthy, A. Is the non-market literature adequate to support coastal and marine management? Ocean Coast Manage, v. 50, n. 5-6, p. 363-378, 2007.

Piedade, F.O. Legislação ambiental e a gestão de unidades de conservação no Maranhão. Rev. Direito Amb. Soc., v. 3, n. 2, p. 175-189, 2015.

Pindyck, R.S.; Rubinfeld, D.L. Microeconomia. Pearson Education do Brasil, 742 p., São Paulo, 2013.

Rassweiler, A.; Costello, C. \& Siegel, D.A. Marine protected areas and the value of spatially optimized fishery management. Proc. Nat. Acad. Sci., v. 109, n. 29, p. 11884-11889, 2012.

Reid-Grant, K. \& Bhat, M.G. Financing marine protected areas in Jamaica: An exploratory study. Mar. Policy, v. 33, n. 1, p. 128-136, 2009.

Remoundou, K.; Koundouri, P.; Kontogianni, A.; Nunes, P. A. \& Skourtos, M. Valuation of natural marine ecosystems: an economic perspective. Environ. Sci. Pol., v. 12, n. 7, p. 10401051, 2009.

Rietbergen-McCracken, J. \& Abaza, H. Environmental valuation: a worldwide compendium of case studies. Routledge, 256 p., Abingdon, 2013.

Salani, S.; Lotufo, T.M.C.; Hajdu, E. Sigmaxinella cearense sp. nov. from sandstone reefs off Fortaleza (Ceará State, Brazil) (Desmacellidae, Mycalina, Poecilosclerida, Demospongiae). Zootaxa, v. 1369, p. 43-53, 2006.

Santos-Corrêa, S. R. Valoração econômica dos recursos ambientais do Parque Municipal da Boa Esperança, Ilhéus, e da Reserva Particular do Patrimônio Natural Serra do Teimoso, Jussari, Bahia. Dissertação de Mestrado, Programa de Pós-graduação em Desenvolvimento Regional e Meio Ambiente, Universidade Estadual de Santa Cruz, 81 p., Ilhéus, 2005.

Saout, S.L.; Hoffmann, M.; Shi, Y.; Hughes, A.; Bernard, C.; Brooks, T.M.; Bertzky, B.; 
Butchart, S.H.M.; Stuart, S.N.; Badman, T. \& Rodrigues, A.S.L. Protected Areas and Effective Biodiversity Conservation. Science, v. 342, n. 6160, p. 803-803, 2013.

Seppelt, R.; Dormann, C. F.; Eppink, F. V.; Lautenbach, S. \& Schmidt, S. A quantitative review of ecosystem service studies: approaches, short comings and the road ahead. $J$ Applied Ecol., v. 48, n. 3, p. 630-36, 2011.

Silva Jr, J. M. Parque nacional marinho de Fernando de Noronha: uso público, importância econômica e proposta de manejo, pp. 335-442, in Anais do II Simpósio de Áreas Protegidas no Âmbito do Cone Sul, Pelotas, 2003.

Silva, A.G.D. Valoração econômica ambiental em unidades de conservação: um panorama do contexto brasileiro. Dissertação de Mestrado, Programa de Pós-graduação em Ciências da Engenharia Ambiental, Universidade de São Paulo, 118 p., São Paulo, 2015.

Sissini, M.N. Hapalidiaceae (Corallinophycidae, Rhodophyta) no litoral brasileiro - diversidade e biogeografia. Dissertação de Mestrado, Programa de Pós-graduação em Biologia de Fungos, Algas e Plantas, Universidade Federal de Santa Catarina, 147 p., Florianópolis, 2013.

Soares, M.O.; Paiva, C.C.; Freitas, J.E.P. \& Lotufo, T.M.C. Gestão de unidades de conservação marinhas: o caso do Parque Estadual Marinho da Pedra da Risca do Meio, NE - Brasil. Rev. Gestão Costeira Integr., v. 11, n. 2, p. 257-268, 2011.

Soares, M.O.; Rossi, S.; Martins, F.A.S. \& Carneiro, P.B.M. The forgotten reefs: benthic assemblage coverage on a sandstone reef (Tropical South-western Atlantic). J. Mar. Biol. Assoc. U. K., 2016. DOI:10.1017/S0025315416000965

Thur, S.M. User fees as sustainable financing mechanisms for marine protected areas: an application to the Bonaire National Marine Park. Mar. Policy, v. 34, n. 1, p. 63-69, 2010.

Trujillo, J.C.; Carrillo, B.; Charris, C.A. \& Velilla, R.A. Coral reefs under threat in a Caribbean marine protected area: assessing divers' willingness to pay toward conservation. Mar. Policy, v. 68, p. 146-154, 2016.

Vitali, M. Conservação da biodiversidade e uso dos recursos naturais em Fernando de Noronha: sustentabilidade em ambientes sensiveis. Dissertação de Mestrado, Programa de Pós-graduação em Desenvolvimento Sustentável, Universidade de Brasília, Brasília, 2010.

Wallmo, K. \& Edwards, S. Estimating public values for marine protected areas in the northeast united states: a latent class modeling approach. NOAA, 72 p., 2007.

Zar, J.H. Biostatistical analysis. 5 ed. Prentice Hall, New Jersey, 2010.

Zhongmin, X.; Guodong, C.; Zhiqiang, Z.; Zhiyong, S. \& Loomis, J. Applying contingent valuation in China to measure the total economic value of restoring ecosystem services in Ejina region. Ecol. Econ., v.44, n.2-3, p.345-358, 2003. 


\begin{abstract}
ANEXO
Questionário aplicado para estimar a disposição a pagar pela preservação do PARQUE ESTADUAL MARINHO DA PEDRA DA RISCA DO MEIO
\end{abstract}

Caro respondente,

Você está participando de um PROJETO DE PESQUISA do Curso de Pós-Graduação em Ciências Marinhas Tropicais da Universidade Federal do Ceará (UFC), que tem como finalidade valorar o Parque Estadual Marinho da Pedra da Risca do Meio. Responda o questionário de forma mais sincera. Não é necessário se identificar.

1: Gênero: ( ) Feminino ( ) Masculino

2: Idade: anos

3: Grau de instrução:

$\begin{array}{llllll} & \text { Fundamental } & \text { Médio } & \text { Superior } & \text { Mestrado } & \text { Doutorado } \\ \text { Incompleto } & (\text { ) } & (\text { ) } & (\text { ) } & (\text { ) } & (\text { ) } \\ \text { Completo } & (\text { ) } & (\text { ) } & (\text { ) } & (\text { ) } & (\text { ) }\end{array}$

4: Profissão

5: Renda: ～( ) R $\$ 0,00-\mathrm{R} \$ 750,00$

( ) $\mathrm{R} \$ 751,00-\mathrm{R} \$ 1500,00$

( ) $\mathrm{R} \$ 1501,00-\mathrm{R} \$ 5000,00$

( ) Acima de $\mathrm{R} \$ 5000,00$

6: Você conhece o Parque Marinho? ( ) Sim ( ) Não

6.1: Você já foi ao Parque Marinho? ( ) Sim ( ) Não

6.2: Qual atividade você realizou no Parque Marinho?
( ) Mergulho
( ) Pesca Comercial
( ) Pesca Esportiva
( ) Outras:

7: Você tem interesse na preservação do Parque Marinho? ( ) Sim ( ) Não

8: Imaginemos uma situação em que, para que o Parque Marinho continuasse a existir como está hoje, as pessoas tivessem que pagar algum valor. VOCÊ estaria disposto a pagar que quantia POR MÊS pela manutenção e conservação do Parque?

9: Caso NÃO esteja disposto a pagar, qual motivo?

( ) Não possui recurso suficientes

( ) Não tem por que pagar, é obrigação do Governo do Estado

( ) O dinheiro arrecadado pode ser usado para outros fins

( ) Não tenho interesse 